\section{Die Rheumatologie im Wandel}

O $\mathrm{ft}$ denke ich an meine Weiterbildungszeit zum Rheumatologen zurück. Die medikamentösen Mittel beschränkten sich im Wesentlichen auf Gold, Sulfasalazin und Methotrexat. Wenn das nicht half, schickten wir die Rheumakranken in eine Rehaklinik, wo sie lernen sollten, mit ihren Defiziten zurechtzukommen. Ich sah damals unzählige Patienten mit deformierten Gelenken; bei diesen diskutierten wir, ob ein rheumachirurgischer Eingriff ihr Leiden wohl ein wenig lindern könnte.

Im Vergleich dazu haben wir heute paradiesische Zustände: Um die Jahrtausendwende kam Leflunomid auf den Markt und erweiterte unser therapeutisches Spektrum; das war aber nur ein kleiner Schritt im Vergleich zu dem Meilenstein, den die Einführung der biologischen Therapeutika bedeutete. TNF-Inhibitoren machten den Anfang; im Laufe weniger Jahre folgte eine ganze Batterie von Biologika, die verschiedenste Angriffspunkte im Fokus hatten. Somit können wir heutzutage auch $\mathrm{Pa}$ tienten helfen, bei denen das Prinzip der
TNF-Inhibition nicht oder ungenügend wirkt.

Wir haben Rheuma nicht besiegt, denn heilen können wir die Erkrankung immer noch nicht. Aber wir können neu erkrankte Patienten beruhigen und ihnen auf den Weg geben, dass sie "nicht im Rollstuhl landen werden“. Vielmehr werden sie ein ganz normales Leben führen können.

Die Rheuma-Rehakliniken klassischer Prägung gibt es kaum noch - die ambulanten Therapiemöglichkeiten und deren Erfolge sind so gut, dass eine Rehabilitation nur selten nötig ist. Gleiches gilt für die Rheumachirurgie: $\mathrm{Da}$ die Gelenke nicht mehr zerstört werden, sind rekonstruktive Eingriffe kaum mehr notwendig.

Bei aller Euphorie sollte man aber auch die vorhandenen Probleme sehen: _Es gibt nur noch wenige Spezialisten, die spezielle Rheumaoperationen, zum Beispiel am kraniozervikalen Übergang noch beherrschen.

_-Die neuen Medikamente sind extrem teuer. Damit ergibt sich für den Arzt

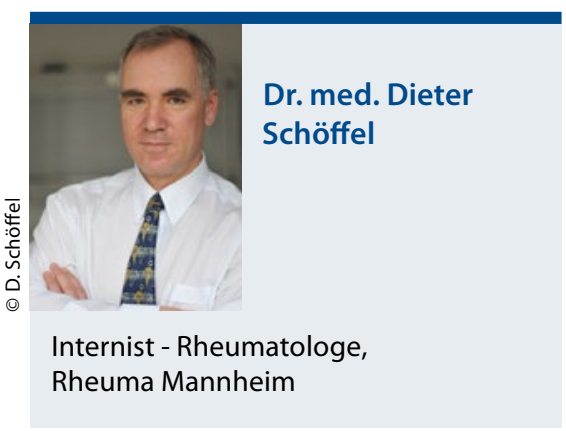

immer das Risiko, möglicherweise selbst mit seinem Vermögen für die verordneten Therapien haften zu müssen.

_Es gibt viel zu wenige Rheumatologen. Die Möglichkeit, Patienten durch eine Frühtherapie ihrer rheumatoiden Arthritis in Remission zu bringen, scheitert oft daran, dass der Patient erst viele Monate später einen Vorstellungstermin bei einem Rheumatologen bekommt.

Unter dem Strich aber ist das Fazit positiv. Wir können glücklich sein über die neuen Möglichkeiten in der Rheumatologie: Wir Ärzte, vor allem aber die betroffenen Patienten.

\title{
Die Orthopädie-Praxis im Jahr 2037
}

$\mathrm{B}_{\mathrm{A}}^{\mathrm{l}}$ lickt man unter medizinrechtlichen Aspekten auf die Strukturen unseres Gesundheitswesens der letzten 20 Jahre zurück, hat sich Wesentliches geändert. Aus der Beobachtung dieser Änderungen sei ein Ausblick auf die Medizin im Jahr 2037 gewagt.

Die meines Erachtens richtungsweisende Zäsur leitete das GKV-Modernisierungsgesetz ein. Seit 2004 ist es Kliniken gestattet, Medizinische Versorgungszentren (MVZ) zu gründen und dadurch in den ambulanten Bereich einzudringen. Es wäre übertrieben zu behaupten, dass heute - knapp 15 Jahre später - die Klinik-MVZ den „Durchbruch“ geschafft hätten und mit den Vorzügen einer arztbetriebenen Praxis ernsthaft konkurrieren könnten. Tatsächlich steigt aber die Zahl der KlinikMVZ unaufhaltsam. Die Gründe hierfür sind vielfältig: So verließen Ärzte früher die Klinik, um eine Praxis zu gründen

oder zu kaufen. Diese Spezies scheint vom Aussterben bedroht. Angst vor dem unternehmerischen Risiko und unzählige weitere Ängste kennzeichnen die Ärzte von heute. Viele Jungärzte entscheiden sich für ein MVZ, um Familie und Beruf besser verbinden zu können (Stichwort: „work life balance“).

Im Gegensatz zu früher liegt die Leitung eines MVZ in den Händen qualifizierter Manager. Sie treffen strategische Entscheidungen und bauen professionelle Strukturen auf mit dem Ziel, die Fallzahlen der Klinik zu steigern. Die Fachgruppen Orthopädie und Chirurgie sind bedarfsplanerisch nach (Land-)Kreisen aufgeteilt. Der „Kauf“ von Zulassungen durch Kliniken bezweckt häufig eine Monopolstellung. Andere Ärzte werden vom Markt ausgeschlossen.

Die Verlagerung der ambulanten Medizin hin zu den Kliniken ist in vollem Gang. Die orthopädische Praxis 2037

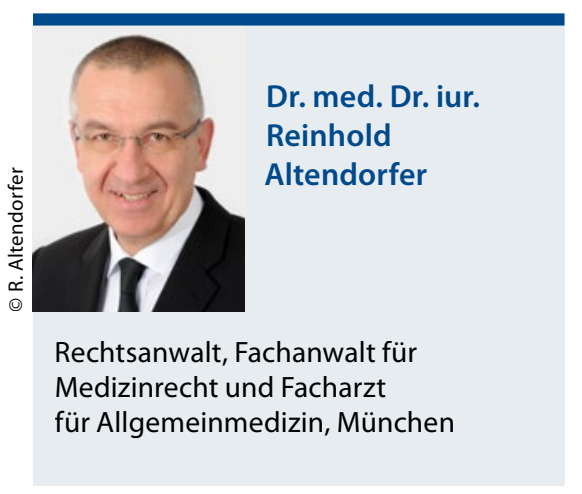

wird eine Einrichtung mit fachlich subspezialisierten Ärzten und einer suffizienten Infrastruktur sein. Röntgen, CT und MRT müssen unter einem Dach ebenso vorgehalten werden wie etwa Physiotherapie und andere flankierende Disziplinen. Nur diese „orthopädische Boutique“, idealerweise an einem krankenhausnahen Standort, wird in 20 Jahren gegen die professioneller werdenden Klinikeinrichtungen bestehen können. 